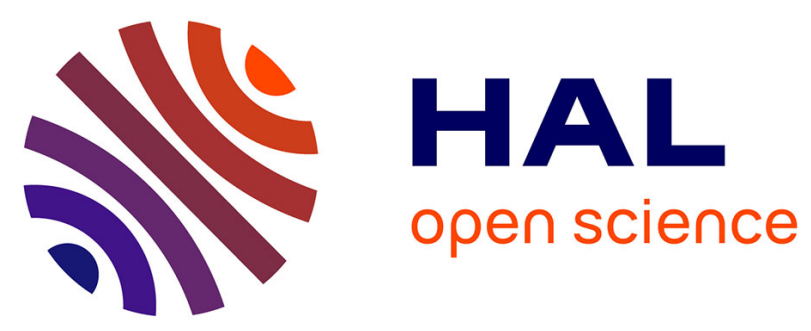

\title{
Une approche socio-historique pour l'étude spatiale des sciences
}

\author{
Michel Grossetti, Béatrice Milard, Marion Maisonobe
}

\section{To cite this version:}

Michel Grossetti, Béatrice Milard, Marion Maisonobe. Une approche socio-historique pour l'étude spatiale des sciences. Histoire de la recherche contemporaine: la revue du Comité pour l'histoire du CNRS , 2015, La recherche scientifique: objet d'étude et enjeu social, 4 (2), pp.142-151. 10.4000/hrc.1105 . halshs-01408371

\section{HAL Id: halshs-01408371 https://shs.hal.science/halshs-01408371}

Submitted on 4 Dec 2016

HAL is a multi-disciplinary open access archive for the deposit and dissemination of scientific research documents, whether they are published or not. The documents may come from teaching and research institutions in France or abroad, or from public or private research centers.
L'archive ouverte pluridisciplinaire HAL, est destinée au dépôt et à la diffusion de documents scientifiques de niveau recherche, publiés ou non, émanant des établissements d'enseignement et de recherche français ou étrangers, des laboratoires publics ou privés. 


\section{Une approche socio-historique pour l'étude spatiale des sciences}

Michel Grossetti, Béatrice Milard, et Marion Maisonobe. 2015. «Une approche sociohistorique pour l'étude spatiale des sciences ». Histoire de la recherche contemporaine. La revue pour le Comité pour l'histoire du CNRS. 4 (2), pp. 142-151

\section{Résumé}

A rebours de l'idée d'une activité scientifique détachée du sol, du milieu dans lequel elle se déploie, l'approche socio-historique des sciences examine la configuration spatiale des activités scientifiques à différentes échelles géographiques : villes, régions, pays et monde. Le développement de cette ligne de recherche accompagne l'émergence de la géographie des sciences, un domaine qui n'est pas encore vraiment établi en tant que spécialité, comme peuvent l'être la sociologie ou l'histoire des sciences, mais qui acquiert une certaine consistance depuis quelques années. Dans ce nouveau paysage, plusieurs orientations se dessinent dont nous donnons un aperçu d'ensemble avant de revenir sur le développement de l'approche socio-historique que nous portons. Cette dernière se fonde sur des données issues d'analyses bibliométriques et d'enquêtes empiriques. .

Mots clés : socio-histoire des sciences, géographie des activités scientifiques, scientométrie spatiale, ligne de recherche

\section{Abstract}

Contrary to the idea that scientific activities are disconnected from the specific environment they are evolving into, the socio-historical approach of science examine the spatial organization of scientific activities at several scales: city-scale, country-scale, and worldscale. The development of this research front go hand in hand with the emergence of geography of science which is not yet a deeply rooted specialty, like sociology or history of science are, but which acquired a certain consistency in recent years. In this new landscape, different directions are available. We present them before focusing on the trajectory of the socio-historical approach we promote. Our approach is empirically based on bibliometric data and benefit from the support of spatial analytical tools such as maps.

Key words: socio-history of science, geography of scientific activities, spatial scientometrics, research front

Michel Grossetti:

Directeur de recherche au CNRS et directeur d'études à l'EHESS. Ses travaux portent sur les réseaux sociaux, l'innovation et l'histoire des institutions scientifiques.

Sur la question de l'organisation spatiale des activités scientifiques, il a publié entre autres les ouvrages Université et territoire. Toulouse, un système local d'enseignement supérieur (Presses universitaires du Mirail, 1994), Science, industrie et territoire (Presses universitaires du Mirail, 1995) et a dirigé avec Philippe Losego La territorialisation de l'enseignement 
supérieur et de la recherche. France, Espagne, Portugal (L'Harmattan, 2003). Il a dirigé avec Denis Eckert le programme ANR Geoscience.

Béatrice Milard :

Professeure de sociologie à l'université de Toulouse 2 (LISST-Cers). D'une manière générale, ses travaux portent sur les écrits publiés et leur implication dans la dynamique des relations sociales et des collectifs de travail. Sur la territorialisation de la recherche, elle a publié plusieurs articles et chapitres de livre qui, à partir d'analyses bibliométriques, mettent en évidence les principales évolutions spatiales de ce secteur d'activité dans plusieurs pays.

Marion Maisonobe

Doctorante en géographie au LISST-CIEU (CNRS et université de Toulouse). Sa thèse porte sur la géographie des activités de publications scientifiques dans le monde. Membre du groupe FMR (flux, matrices, réseaux) et du secrétariat de rédaction de la revue Netcom, elle s'intéresse aux réseaux scientifiques, à la diffusion spatiale des innovations et à l'émergence de communautés internationales. Ses premiers travaux sont parus en 2013 dans la revue de géographie M@ppemonde et dans DNA Repair, revue spécialisée en biologie moléculaire.

L'objectif de cet article est de présenter une ligne de recherche sur l'organisation spatiale des activités scientifiques. Il ne prétend pas faire un inventaire complet des recherches conduites sur cette question, en France ou au niveau international, même si nous évoquerons un certain nombre d'entre elles. Nous avons contribué à divers titres au développement de cette ligne de recherche et c'est de ce point de vue d'acteurs du domaine que nous avons écrit ce texte.

Nous commencerons par un rapide état des travaux menés depuis une cinquantaine d'années sur la dimension géographique des activités scientifiques. Nous verrons que, si les études sociales sur les sciences ont connu un développement considérable depuis le début des années 1970, l'analyse spatialisée des activités de recherche académique est restée en retrait et que la géographie des sciences n'est pas encore vraiment établie en tant que spécialité, comme peuvent l'être la sociologie ou l'histoire des sciences. En revanche, des travaux inscrits dans des domaines divers et peu reliés entre eux ont abordé la question spatiale. Ensuite, nous nous centrerons sur la ligne de recherche à laquelle nous avons contribué, une ligne qui associe analyses historiques, sociologiques et géographiques.

\section{Un stade embryonnaire pour les études spatiales des sciences?}

Le domaine où les réflexions de type géographique sur l'activité scientifique sont les plus élaborées est celui des recherches historiques. C'est déjà dans ce domaine que le sociologue des sciences Joseph Ben-David avait amorcé une réflexion sur les différences entre les contextes nationaux d'exercice de la recherche (Ben-David, 1968). C'est aussi dans une perspective historique qu'Harold Dorn, un élève de Thomas Kuhn, s'est lancé il y a quelques années dans un essai sur le lien entre les conditions naturelles et les besoins scientifiques des sociétés (à partir des débats sur le «mode de production asiatique »de Marx), curieusement titré The geography of science (Dorn, 1991). Mais c'est surtout dans les années récentes que des géographes historiens et des historiens des sciences ont développé une analyse ancrée dans les science studies, notamment à partir de certaines réflexions de l'historien Steven Shapin («si, comme l'établit avec certitude la recherche empirique, la science est un produit local, comment est-ce qu'elle voyage avec ce qui semble être une si 
remarquable efficacité ? », Shapin, 1995, p. 307). Steven J. Harris s'est intéressé au fonctionnement de la Compagnie de Jésus pour s'essayer à une "géographie de la connaissance» (Harris, 1998). David Livingstone a cherché à conceptualiser une " géographie des connaissances scientifiques » (Livingstone, 2003). D'autres auteurs se sont plutôt inspirés des réflexions de Wallerstein sur le système-monde pour entreprendre une analyse « géohistorique » des déplacements des scientifiques au cours de leur carrière (Taylor, Hoyler, Evans, 2008). Dans des articles de synthèse, l'accent est mis sur la nécessité de dépasser une analyse des lieux et des cultures pour aller vers une étude des flux et des déplacements, tout en insistant, selon la rhétorique habituelle pour établir un nouveau domaine, sur l' « évidence » du «tournant géographique » des science studies (Naylor, 2005 ; Powell, 2007 ; Besse, 2010 ; Eckert et Baron, 2013). Cette «section géographique »des sciences studies, en cours de constitution, reste surtout composée d'analyses historiques, malgré l'intéressante tentative de l'un de ces géographes historiens pour suivre par l'observation ethnographique un projet de recherche dans l'Arctique canadien (Powell, 2009). Une géographie des spécialisations scientifiques commence néanmoins à exister en France qui présente également une dimension plus quantitative (Cuyala 2013, Maisonobe 2013).

Pour le moment, on trouve peu de géographes utilisant des données quantitatives pour produire des analyses spatiales de la science académique actuelle et de ses évolutions. La française Madeleine Brocard s'y est essayée pour sa thèse à la fin des années 1970, et de nouveau pour un atlas de la science au début des années 1990 (Brocard, 1978, 1991), mais elle s'est éloignée depuis lors de cet objet de recherche. Si l'on veut comprendre l'inattention des géographes pour cet objet, il faut savoir que même si la récolte de données scientométriques existe depuis plus d'un siècle, les organismes officiels de la statistique publique n'y ont eu recours que tardivement. Le manque de données administratives disponibles est souligné à plusieurs reprises par Brocard dans son travail sur la recherche française. A partir des années 1950, quelques organismes gouvernementaux enregistrent des données sur l'investissement économique et les ressources humaines dans la recherche et le développement (input) ; mais jusqu'aux années 1980, mesurer la productivité scientifique et la visibilité des travaux de recherche (output) n'intéresse pas l'administration (Godin, 2002). Pour Benoît Godin, les administrations se focalisent essentiellement sur les mesures d'input jusqu'aux années 1980 en raison de l'influence de l'Organisation de Coopération et de Développement Economique (OCDE). Les recommandations de l'OCDE pour la mesure des intrants de la recherche sont consignées depuis 1963 dans un document appelé le Manuel de Frascati.

A partir des années 1990, accompagnant les nouvelles orientations des politiques scientifiques, les travaux sur l'innovation en économie régionale et urbaine font une place croissante aux débouchés des investissements publics et privés dans la recherche et le développement. L'activité scientifique est progressivement perçue comme productrice de richesse et moteur de compétitivité urbaine. Parmi les recherches participant de cette tendance, il faut souligner le travail de Christian Matthiessen et Annette Schwarz, de l'institut de géographie de Copenhague, qui utilisent des données bibliométriques à l'échelle des villes pour comparer la production scientifique d'une série de grandes agglomérations (Matthiessen et Schwarz, 1999), ainsi que celui de Heike Jöns à l'université d'Heidelberg, qui a entrepris une véritable réflexion sur l'organisation spatiale des activités scientifiques (Jöns, 2007). Dans le cadre de la géographie, ce type d'analyse reste tout de même exceptionnel jusqu'à la fin des années 2000.

Ces analyses sont plutôt réalisées dans des spécialités proches, comme la géographie de l'innovation par exemple, qui est un domaine assez bien établi, regroupant des contributions issues principalement de la géographie économique et de l'économie régionale ou industrielle (Feldman et Massard, 2002, Autant-Bernard et alii, 2007). Il s'agit en particulier de mettre en 
évidence la dimension spatiale des liens entre la recherche académique et l'industrie et les effets de la R\&D sur le développement économique local, ce qui implique parfois d'analyser le déploiement dans l'espace de la recherche académique (Sterlacchini, 2008). On trouve aussi des analyses spatiales plus centrées sur la recherche académique dans les études sur les sciences et les technologies, sans qu'une approche géographique soit pour autant revendiquée. La dimension internationale des activités scientifiques en particulier fait l'objet d'une abondante littérature de la part de scientifiques ${ }^{1}$, d'économistes ou de spécialistes des relations internationales (Weiss, 2005), et même de sociologues (Vinck, 1996, Gingras, 2002). Certains travaux se centrent sur les hiérarchies internationales ou les phénomènes de brain drain, brain back, migration pendulaire et diaspora scientifique (Meyer, Kaplan, Charum 2001). Il existe aussi une littérature sur les activités scientifiques dans les pays «en développement » ou «non hégémoniques »(Gaillard, Krishna et Waast, 1997 ; Losego et Arvanitis, 2008) et des travaux sur la division internationale du travail scientifique (Kreimer et Lugones, 2003). On trouve enfin des réflexions sur les politiques nationales ou européennes de recherche dans la compétition internationale (Laredo, 2004), sur la construction d'un espace européen de la recherche (Hoekman, Frenken, Oort 2008), sur l'équilibre global entre macro-régions mondiales (Glänzel, Debackere, Meyer, 2008), sur la tension entre les espaces nationaux et la science mondiale (Mallard, Paradeise, Peerbaye, 2008). On trouve beaucoup moins de travaux sur le niveau infra-national, même si certains chercheurs s'efforcent de repérer à partir de données bibliométriques des effets de déconcentration en Europe (Zitt, Barré, Sigogneau, Laville, 1999) ou en Chine (Wei, 2008). Il existe également des travaux sur les dynamiques locales qui expliquent des trajectoires de constitution de pôles (Bozeman, Laredo et Mangematin, 2007). Enfin, parmi toute la littérature scientométrique qui présente pourtant souvent ses résultats selon des critères géographiques (avec des visualisations et cartographies parfois très élaborées), on ne trouve que quelques rares travaux problématisant la question de l'espace.

C'est dans ce contexte global, foisonnant mais peu organisé, que nous avons développé une approche socio-historique dont la particularité est d'associer en permanence les analyses quantifiées de l'organisation spatiale des activités scientifiques (pour mettre en évidence des situations et des processus que l'on cherche ensuite à expliquer), l'histoire des institutions scientifiques (qui rend compte globalement des écarts entre la «carte » scientifique et les «cartes » politiques, économiques ou démographiques), et la sociologie des sciences (qui permet d'analyser les logiques sociales impliquées tant dans le passé des institutions que dans le présent de l'activité scientifique ordinaire).

\section{Des relations science-industrie à l'étude spatiale des sciences}

Cette ligne de recherche a débuté comme une question annexe d'un programme consacré aux aspects géographiques des relations science-industrie par l'un d'entre nous à partir de 1988 (voir Grossetti, 1995 pour une synthèse des résultats de ce programme). Ces recherches mettaient en évidence l'existence d'importantes différences quantitatives et qualitatives entre les institutions scientifiques (laboratoires, instituts de formation) des différentes villes françaises. Ces différences ne sont pas réductibles à des effets de structure urbaine (taille des villes, compositions des populations) ou de spécialités industrielles. Elles sont le fruit d'une longue histoire. Il existe donc une «carte» spécifique des activités

\footnotetext{
${ }^{1}$ Par exemple : Ramamurti Shankar, «Globalization and Science: A Speeded-Up Virtuous Cycle », YaleGlobal, 28 mars 2003 ; Sealy Cordelia, "Globalization of science », Materials Today, Volume 7, n5, mai 2004, p. 1, Elsevier Ltd.
} 
d'enseignement supérieur et de recherche, issue d'un processus historique complexe. Étudier ce processus permet de problématiser l'ambiguïté de la science, censée produire des savoirs universels à partir d'organisations toujours dépendantes de contextes locaux ou nationaux, ce qui implique toujours simultanément pour les scientifiques l'insertion dans des réseaux d'échanges nationaux et internationaux (participation aux colloques, publications, etc.) et dans des communautés locales (l'université comme lieu de vie communautaire, le laboratoire comme entité humaine et scientifique à la fois, etc.). A ainsi débuté une nouvelle forme de socio-géographie des activités scientifiques.

L'échelle spatiale infranationale pertinente est celle de la ville. L'examen de la répartition des institutions d'enseignement supérieur et de recherche en France montre qu'elles se concentrent pour l'essentiel dans les grandes villes. Les pôles scientifiques sont donc associés à des villes qu'ils contribuent à spécifier. Chacun des systèmes scientifiques locaux est particulier, par les caractéristiques des organisations (multiples en France) qui le composent, par les liens qui s'établissent entre elles, par son histoire. Toutefois, il existe parfois des similarités fortes entre certains d'entre eux. Par exemple, Toulouse, Grenoble et Nancy ont des systèmes scientifiques relativement semblables au moins sur le plan des disciplines d'ingénierie avec la présence de nombreuses écoles d'ingénieurs dépendant de l'Éducation nationale et rassemblées en instituts nationaux polytechniques, et avec de nombreux chercheurs en sciences de l'ingénieur.

Le constat de certaines de ces similarités, celles qui concernent Grenoble et Toulouse en particulier, nous a incités à rechercher leur origine, puis de proche en proche à reconstituer une sorte de schéma général de la construction spatiale du système français d'enseignement supérieur et de recherche. Ce travail s'est appuyé sur des travaux existants d'historiens et sur un travail spécifique d'histoire orale ${ }^{2}$. Son principal apport (outre des analyses spécifiques au cas toulousain) est de mettre en relation les orientations prises par les facultés des sciences au début du siècle (analysées notamment par André Grelon) avec leur plus ou moins grande précocité dans le développement de certaines disciplines appliquées au cours des années 1945 - 1968, puis avec leur situation actuelle.

Sur cette base a été conduite une étude financée par le Plan Urbain et le Ministère de 1'Education Nationale sur le thème « Ville et université ». Fatima Ayat, Martine Azam, JeanPaul Laurens et Patricia Vannier ont contribué à ce travail, dont est issu l'ouvrage Université et Territoire. Un système scientifique local, Toulouse et Midi-Pyrénées (Presses Universitaires du Mirail, 1994, avec des contributions de Michel Idrac et Daniel Filâtre). Cet ouvrage comprend une partie historique et une partie statistique sur la situation d'un "système scientifique local », celui de Toulouse. L'idée centrale est de traiter l'ensemble des établissements d'enseignement supérieur et de recherche d'une même agglomération urbaine, d'en faire l'histoire, d'en caractériser la situation contemporaine et de mettre en évidence leurs interdépendances.

\section{Villes et institutions scientifiques à différentes échelles spatiales}

Le cas de Toulouse étant bien documenté, il s'agissait de vérifier si l'on pouvait trouver des processus similaires dans d'autres villes françaises. Michel Grossetti s'est associé avec André Grelon et divers autres chercheurs, historiens, géographes et sociologues (dont Béatrice Milard, alors doctorante) pour l'étude historique «Villes et institutions scientifiques »

\footnotetext{
2 Michel Grossetti a bénéficié à la fin des années 1980 d'un petit financement d'un programme interdisciplinaire du CNRS, le PIRTTEM, pour réaliser une étude comparative de l'histoire des laboratoires de sciences pour l'ingénieur à Grenoble, Nancy et Toulouse. Il a par la suite approfondi le cas de l'informatique avec Pierre-Eric Mounier-Kuhn et celui du génie chimique avec Claude Detrez.
} 
(financée par le PIR-Villes). En réalisant cinq monographies locales (Montpellier, Toulouse, Nantes, Lille et Nancy) et trois monographies sectorielles (génie chimique, informatique, mécanique des fluides) cette étude constitue une sérieuse mise à l'épreuve du cadre d'analyse esquissé à l'issue des recherches précédentes. Outre la confirmation de certaines hypothèses (importance du rôle des villes dans la mise en place des institutions d'enseignement supérieur et de recherche ; présence de systèmes locaux d'innovation à des périodes relativement anciennes), elle a permis de faire émerger divers résultats nouveaux, concernant le rôle des industriels dans le développement des instituts au début du siècle, et les rapports entre l'État et les collectivités locales et de dégager une périodisation d'ensemble. Sur le premier point, les monographies mettent en avant le rôle dominant des universitaires eux-mêmes comme porteurs des projets, les industriels n'intervenant souvent qu'après coup comme support de l'action, ainsi que l'existence de modèles (Nancy pour la chimie, Grenoble pour l'électricité) diffusés par les universitaires. Sur le second, l'étude fait émerger une période intermédiaire, entre 1919 et 1950, au cours de laquelle l'État a repris en mains progressivement les rênes du système d'enseignement supérieur et de recherche. La Troisième République, surtout dans sa première période (avant 1914) a constitué un moment de relative décentralisation des pouvoirs, l'État laissant les collectivités locales et autres acteurs locaux prendre des initiatives importantes qui ne seront plus possibles par la suite (et surtout après 1945). Le fameux «modèle républicain » caractérisé par Pierre Grémion ne fonctionne pas pour les villes dans cette période : les acteurs locaux ont l'initiative, le préfet est absent du jeu, le recteur intervient un peu mais rarement de façon décisive. Tout se joue entre les universitaires (représentés par leurs doyens), les élus municipaux ou départementaux et le ministère. ${ }^{3}$.

Le programme européen INTERREG-II-C a été ensuite l'occasion de commencer des comparaisons internationales (Grossetti, Losego et Milard, 2002; Grossetti et Losego, eds, 2003). Il s'agissait d'étudier les cartes scientifiques de la France, de l'Espagne et du Portugal (le «Sud-ouest européen »), en associant une analyse de leur constitution historique, une étude statistique des publications scientifiques par département et par région, et enfin une étude approfondie de la situation des antennes universitaires françaises. On y montre que l'histoire du développement universitaire des pays détermine en partie la répartition spatiale de la production scientifique au niveau du rythme de déconcentration territoriale comme de l'implantation des spécialités disciplinaires. L'analyse bibliométrique des cosignatures d'articles menée par Béatrice Milard à l'échelle des trois pays a mis en évidence une tendance à l'indépendance des régions de province vis-à-vis des capitales. Mais le maillage territorial des collaborations reste fortement marqué par des effets de proximité et d'inertie et révèle des hiérarchies entre les territoires. Le cas de des antennes universitaires en France illustre ces déséquilibres territoriaux du fait de leur avenir qui apparaissait particulièrement incertain. En 2004, Béatrice Milard et Michel Grossetti ont commencé une recherche sur la Russie en collaboration avec le géographe Denis Eckert et divers collègues de ce pays. Une analyse statistique des publications russes a été réalisée (Milard et Grossetti 2006, paru également en russe). Les résultats ont montré une déconcentration assez similaire à celle que l'on observe dans d'autres pays (baisse de la part relative des publications issues de la capitale). Béatrice Milard a prolongé cette étude en montrant combien l'héritage soviétique continue à marquer l'organisation et les relations entre chercheurs de la nouvelle science russe (Milard 2008).

En 2009, nous avons saisi l'opportunité d'un appel à projet de l'Agence Nationale de la Recherche sur le thème «Sciences, technologies et savoirs en sociétés » pour revenir sur la question de l'organisation spatiale des activités scientifiques. Est ainsi né le projet

\footnotetext{
${ }^{3}$ Les résultats obtenus dans le cadre de cette recherche ont fait l'objet de diverses publications (notamment dans deux ouvrages coordonnés par André Grelon, Des ingénieurs pour la Lorraine et La formation des ingénieurs en électricité et en électrotechnique) mais une grande partie est restée sous la forme d'un rapport (Grossetti et alii, 1996, http://w3.lisst.univ-tlse2.fr/cv/publis/MG4.pdf)
} 
«Geoscience» («Science locale, nationale, mondiale en transformation. Pour une sociogéographie des activités et des institutions scientifiques académiques ») impliquant des sociologues (Michel Grossetti, Béatrice Milard, André Grelon, Dominique Vinck, Yves Gingras, Rigas Arvanitis), des historiens (René Sigrist, Jérôme Lamy, Yves Lequin, Natalia Tikhonov, Irina Gouzevitch) et des géographes (Denis Eckert, Myriam Baron, Laurent Jégou, Marion Maisonobe). Le projet comprenait notamment une étude bibliométrique de l'organisation spatiale des activités scientifiques, fondée sur le contenu du Web of Science (WoS) de Thomson Reuters ®, en 1999-2001 et 2006-2008. Une procédure de géocodage automatisée a été élaborée pour récupérer les coordonnées géographiques des localités désignées dans les adresses des auteurs des publications indexées dans le WoS. Après des opérations de vérification des données, en partie automatisées, les publications pouvaient se répartir dans plus de 18650 localités au niveau mondial. Pour analyser et visualiser l'information au niveau mondial, il est apparu nécessaire d'agréger des localités proches pour obtenir ce que nous avons appelé des « agglomérations scientifiques » (voir schéma et encadré adaptés de Jégou, 2014). Le découpage des agglomérations a été fait en tenant compte de la répartition de la population qui est un critère dont on connaît la valeur sur toute l'étendue du globe terrestre avec une assez bonne précision (Eckert et alii, 2013). En tout, 10730 «agglomérations scientifiques » ont été constituées.

\section{Le traitement géographique de données bibliographiques}

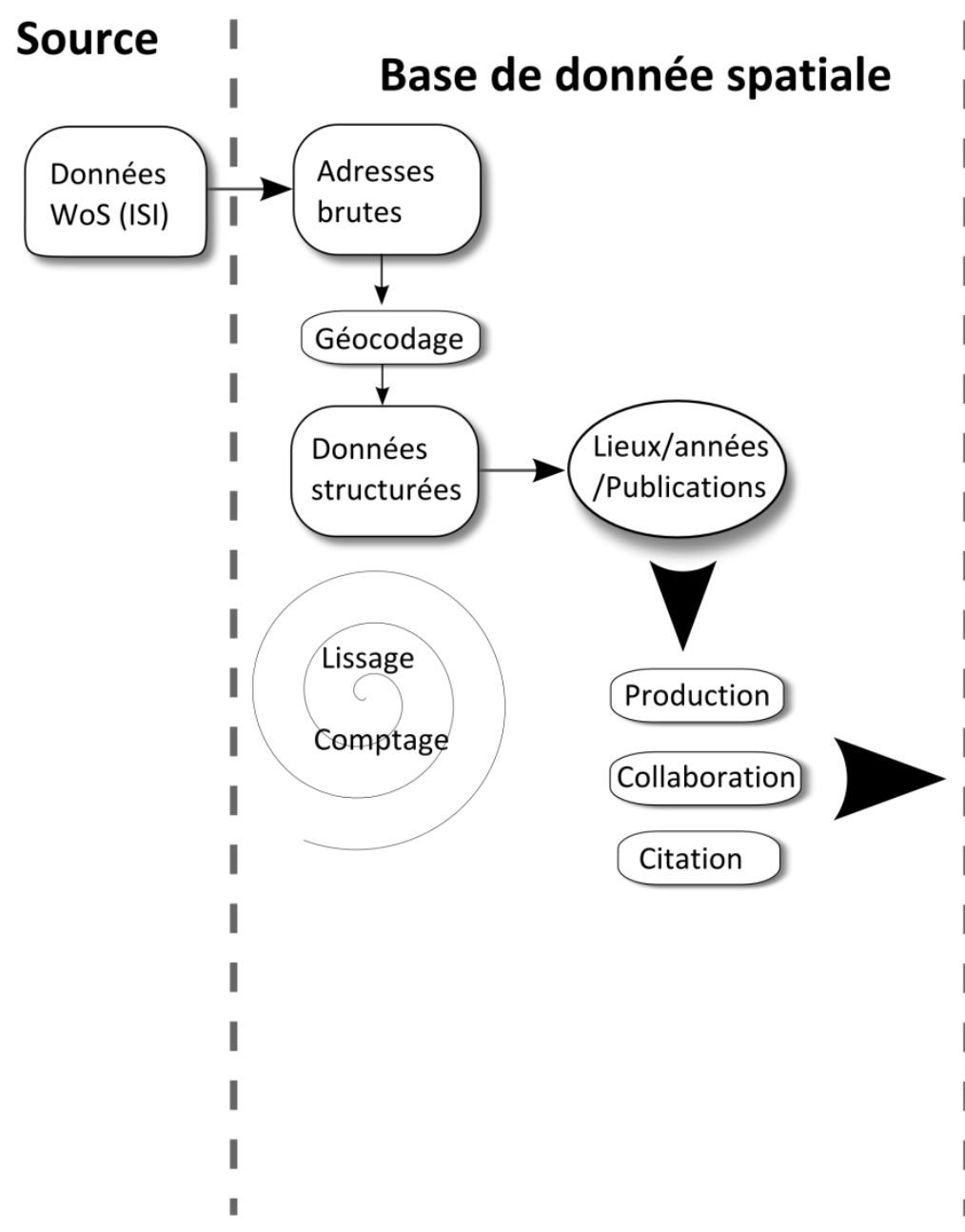

Visualisation des données

I

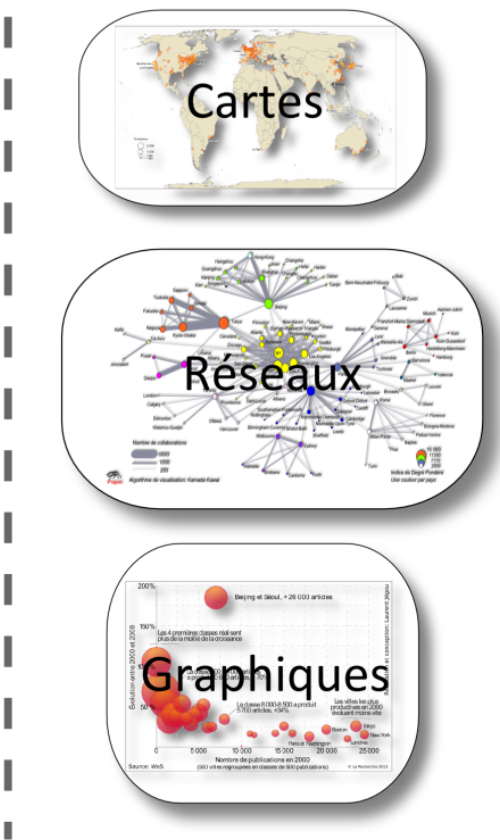

Conception et réalisation: Laurent Jégou et Marion Maisonobe 
A l'issue de ces traitements, nos résultats mettent en évidence un phénomène de rééquilibrage entre pays, au profit de divers pays émergents (Chine, Corée du Sud, Brésil, Turquie, etc.), ainsi que, dans la plupart des pays, une déconcentration des publications au profit d'agglomérations scientifiques secondaires et donc une perte d'hégémonie des capitales et plus grandes villes (Grossetti et alii, 2014). La carte ci-contre témoigne de ce phénomène à l'échelle mondiale..

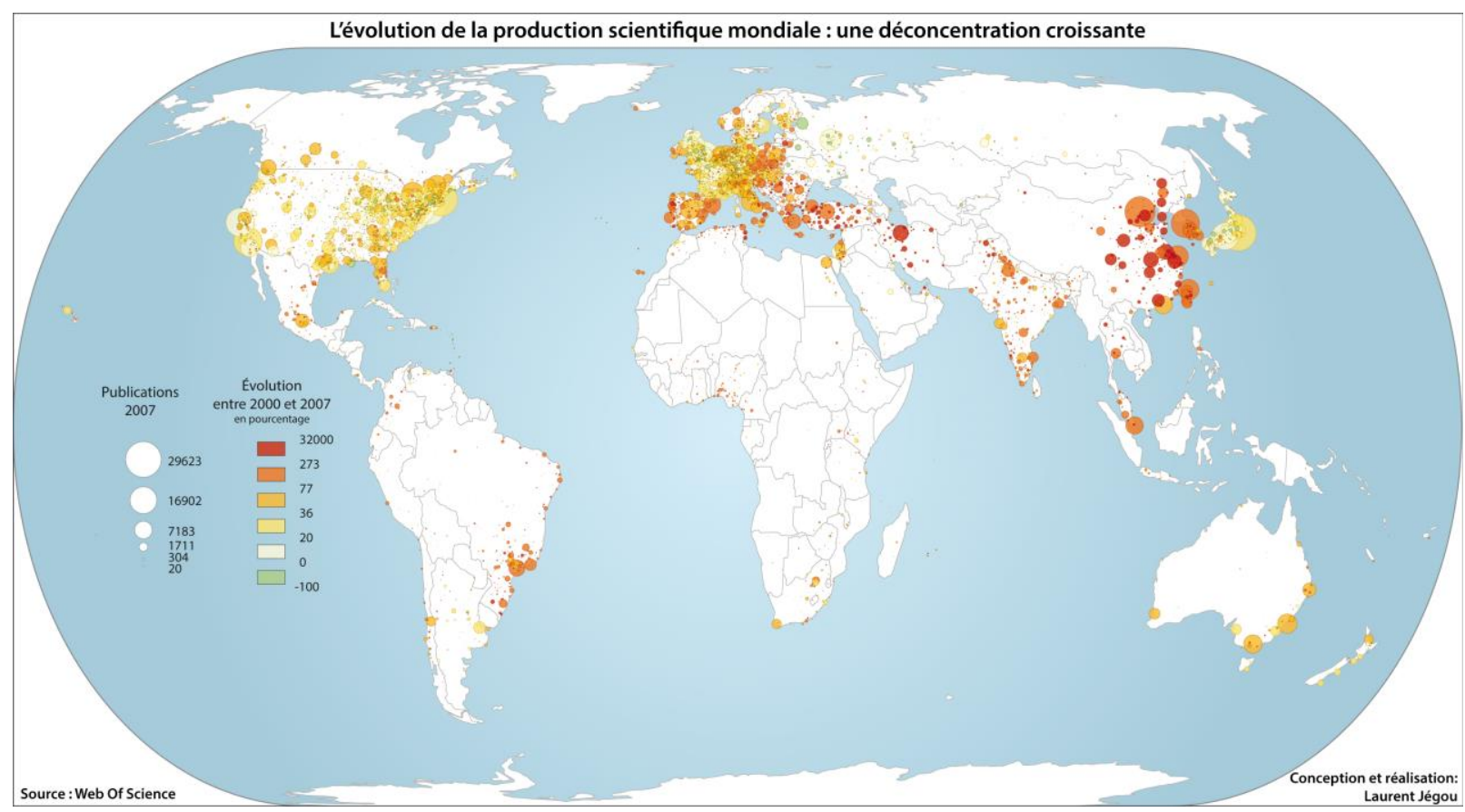

A une échelle plus fine, l'analyse de l'évolution d'une discipline au sein d'une seule région française confirme cette tendance et montre l'importance de la collaboration 
internationale (et les spécialités disciplinaires qui y sont associées) dans le développement et l'autonomisation des pôles secondaires (Milard 2012). Des travaux en cours sur la répartition territoriale des citations des chimistes français entre 1985 et 2005 vont dans le même sens et soulignent que la régionalisation de la recherche n'entraine pas une recherche de moindre qualité (Milard 2014).

Ces résultats confortent les conclusions de travaux menés par une équipe italienne qui a montré que le volume de la production scientifique dans une ville n'est pas «mécaniquement » lié à sa taille (Bonaccorsi et alii, 2005). A partir de très grosses bases de données bibliographiques du Conseil National de la Recherche Italienne ainsi que sur la base de la production scientifique de l'INSERM, ils ont établi qu'on ne trouve pas de corrélation empirique entre une concentration des ressources matérielles et humaines dans une ville et le nombre moyen de publications par chercher dans cette même ville. A un niveau agrégé lissant les différences individuelles et entre disciplines, le nombre de publications est approximativement une fonction linéaire du nombre de personnes ayant des activités de recherche. On observe toutefois des différences en faveur des grands centres scientifiques dans les taux de citations (le nombre moyen de citations reçues par publication), mais cette différence se retreint progressivement (en France par exemple, il n'y en a déjà plus entre l'agglomération parisienne et les grands centres scientifiques de province).

L'idée très répandue de la "masse critique » qui serait nécessaire à la réalisation d'une recherche de qualité n'est donc pas étayée empiriquement. Elle a pourtant fondé de nombreuses politiques de recherche en France ces dernières années, notamment celles destinées à ne promouvoir que quelques pôles d'excellence sur le territoire français. A rebours de l'hypothèse d'un renforcement des hiérarchies (entre les chercheurs, entre les villes, entre les régions), les études empiriques convergent pour mettre en évidence une forme de « démocratisation » des activités scientifiques à toutes les échelles.

\section{Conclusion}

Le développement d'une approche socio-historique pour l'étude spatiale des sciences est particulièrement nécessaire dans un contexte qui voit se multiplier les politiques d' «aménagement du territoire » en matière d'enseignement supérieur et de recherche : regroupements d'universités, initiatives d' « excellence», etc. Il s'agit d'un travail difficile qui nécessite d'associer des analyses historiques, sociologiques, et géographiques dans un cadre d'analyse qui prenne en compte la diversité des échelles spatiales. La compréhension des logiques de diffusion spatiale qui traversent les activités scientifiques et d'enseignement supérieur demande de ne pas se focaliser seulement sur le niveau des nations ou sur le haut des hiérarchies urbaines, mais de prendre en compte tous les niveaux d'organisation spatiale.

\section{Références}

Autant-Bernard Corinne, Mairesse Jacques, Massard Nadine, «Spatial Knowledge Diffusion through Collaborative Networks », Papers in Regional Science, Volume 86, $\mathrm{n}^{\circ} 3$, August 2007, pp. 341-350.

Baron Myriam, «La Formation Supérieure En Régions (France) », Cybergeo: European Journal of Geography, n²79, 2004. 
Ben-David Joseph, Fundamental Research and the Universities: Some Comments on International Differences, Organization for Economic Cooperation and Development, Paris, 1968.

Besse, Jean-Marc, «Approches spatiales dans l'histoire des sciences et des arts ». L'Espace géographique, $\mathrm{n}^{\mathrm{o}} 3$, 2010, pp. 211-24.

Bonaccorsi Andrea, Daraio Cinzia, «Exploring size and agglomeration effects on public research productivity », Scientometrics, Volume 63, n¹, 2005, pp.87-120.

Bozeman Barry, Laredo Philippe, Mangematin Vincent, «Understanding the emergence and deployment of "nano" S\&T », Research Policy, Volume 36, n6, 2007, pp. 807-812.

Brocard Madeleine, «Recherche scientifique et développement régional en France. 1-2 1-2 », thèse de doctorat, 1978.

Brocard Madeleine, La science et les régions : géoscopie de la France, Reclus - La Documentation française, Montpellier - Paris, 1991.

Cuyala Sylvain, «La diffusion de la géographie théorique et quantitative européenne francophone d'après les réseaux de communications aux colloques européens (1978-2011)». Cybergeo: European Journal of Geography, nº 657, 2013.

Dorn Harold, The geography of science, the Johns Hopkins University Press, Baltimore, 1991, 219 p.

Eckert Denis, Baron Myriam, «Construire une géographie de la science », Volume 2, $\mathrm{n}^{\circ} 110$, 2013. http://mappemonde.mgm.fr/num38/intro.html.

Eckert Denis, Baron Myriam, Jégou Laurent, «Les Villes et La Science: Apports de La Spatialisation Des Données Bibliométriques Mondiales », Mappemonde, Volume 110, $\mathrm{n}^{\circ} 2$ <http://mappemonde.mgm.fr/num38/articles/art13201.pdf>

Feldman Maryann P. and Massard Nadine (Eds.), «Institutions and Systems in the Geography of Innovation », in Economics of Science, Technology, and Innovation, Vol. 25, Kluwer Academic Publishers, Norwell, 2002.

Frenken Koen, Sjoerd Hardeman, Jarno Hoekman, « Spatial Scientometrics: Towards a Cumulative Research Program », Science of Science: Conceptualizations and Models of Science, 3, 2009, pp. 222-232

Gaillard Jacques, Krishna Venni Venkata, Waast Roland (éd.), Scientific communities in the developing world. Sage, New Delhi, Thousand Oaks, London, 1997.

Gingras Yves, «Les formes spécifiques de l'internationalité du champ scientifique », Actes de la recherche en sciences sociales, Volume 2, ${ }^{\circ} 141,2002$, pp. 31-45.

Glänzel Wolfgang, Debackere Koenraad , Meyer Martin, « Triad' or 'tetrad'? On global changes in a dynamic world. », Scientometrics, Volume 74, n¹, 2008, pp.71-88. 
Godin Benoît, « Outline for a History of Science Measurement », Science, Technology, \& Human Values, 27, n¹, 2002, pp. 3-27.

Grelon André, Birck Françoise dir., Des ingénieurs pour la Lorraine, XIXe-XXe ss (2e éd. nouvelle préface), Presses universitaires de Nancy, Nancy, 2006, 434 p.

Grelon André, Karvar Anousheh, Gouzevitch Irina, La formation des ingénieurs en perspective. Modèles de référence et réseaux de médiation, XVIIIe-XXe siècles, Rennes, Presses universitaires de Rennes, Coll. Carnot, 2004, 176 p.

Grossetti, Michel, Université et territoire: un système local d'enseignement supérieur, Toulouse et Midi-Pyrénées, Presses universitaires du Mirail, Toulouse, 1994.

Grossetti, Michel, Science, industrie et territoire, Presses universitaires du Mirail, Toulouse, 1995.

Grossetti Michel, Eckert Denis, Jégou Laurent, Gingras Yves, Milard Béatrice, Larrivière Vincent, "Cities and the geographical deconcentration of scientific publications », Urban Studies, Volume 51, n¹0, 2014, pp. 2219-2234.

Grossetti Michel, Grelon André, Birck Françoise, Déré Anne-Claire, Detrez Claude, Emptoz Gérard, Idrac Michel, Laurens Jean-Paul, Mounier-Kuhn Pierre, Milard Béatrice, Canévet Jean-Claude, Marseille Christine, Spiesser Michel, «Villes et Institutions Scientifiques », rapport pour le PIR-VILLES, CNRS, Juin 1996.

Grossetti Michel et Losego Philippe (dir.), La territorialisation de l'enseignement supérieur et de la recherche. France, Espagne, Portugal, L'Harmattan, Paris, 2003.

Grossetti Michel, Losego Philippe et Milard Béatrice, «La territorialisation des activités scientifiques dans le Sud-Ouest européen (France, Espagne, Portugal) », Géographie, Economie, Société, $\mathrm{n}^{\circ} 4,2002$, pp.427-442.

Harris Steven J., «Long-Distance Corporations, Big Sciences, and the Geography of Knowledge », Configurations, Volume 6, n² 2, 1998, pp.269-304.

Hoekman Jarno, Frenken Koen, Oort Frank «The geography of collaborative knowledge production in Europe ». The Annals of Regional Science, Volume 43, n³, 2008, pp. 721-38.

Hong Wei, «Decline of the center: The decentralizing process of knowledge transfer of Chinese universities from 1985 to 2004 », Research Policy, Volume 37, n4, 2008, pp. 580595

Jégou Laurent, «Towards spatially referenced academic data at global scale: the full geocoding of Wos-Datasets, methods and results », 2nd Geography of Innovation International Conference, Utrecht 2014.

En ligne:

http://elio6.eurolio.eu/indico/getFile.py/access?contribId=78\&sessionId=67\&resId=0\&materi $\underline{\text { alId}=\text { paper\&confId }=3}$ 
Jöns Heike, «Transnational mobility and the spaces of knowledge production: a comparison of different academic fields », Social Geography, n², 2007, pp. 79-119

Kreimer Pablo, Lugones Manuel, «Pioneers and Victims: The Birth and Death of Argentina's First Molecular Biology Laboratory », Minerva, n41, 2003, pp.47-69.

Laredo Philippe, «Prospective de l'espace européen de la recherche ». Revue française d'administration publique, 2004, pp. 675-685

Lebeau Boris et Vadelorge Loïc, «Enseignement supérieur, recherche et collectivités locales », Histoire urbaine, 33, 2012, pp. 119-31.

Livingstone David N., Putting Science in its Place: Geographies of Scientific Knowledge, University of Chicago Press, Chicago, 2003.

Losego Philippe, Arvanitis Rigas, «La science dans les pays non-hégémoniques. » Revue d'Anthropologie des Connaissances, Volume 2, n³, 2008, pp. 334-342.

Maisonobe Marion. «Diffusion et structuration spatiale d'une question de recherche en biologie moléculaire », Mappemonde, Volume 2, n¹10, 2013.

Mallard Grégoire, Paradeise Catherine and Peerbaye Ashveen, Global Science and National Sovereignty, Routledge, New-York, 2008.

Matthiessen Christian W., Schwarz Annette W., «Scientific centres in Europe: an analysis of research strength and patterns of specialisation based on bibliometric indicators ", Urban Studies, Volume 36, n³, 1999, pp. 453-477.

Meyer Jean-Baptiste, Kaplan David, Charum Jorge, "Scientific nomadism and the new geopolitics of knowledge / Nomadisme des scientifiques et nouvelle géopolitique des savoirs », International Social Sciences Journal / Revue Internationale des Sciences Sociales, $\mathrm{n}^{\circ} 168$, juin 2001, pp. 341-354.

Milard Béatrice (2008), «L'héritage soviétique dans la nouvelle organisation de la science en Russie. Quels effets sur les pratiques et la valorisation de la recherche ? ", Revue d'Anthropologie des connaissances, vol. 2-3, n 3 , pp. 391-412.

Milard Béatrice (2012), "Emergence, internationalisation et autonomisation de villes scientifiques secondaires. L'exemple de la chimie à Nantes, Angers et Le Mans », in M. Mespoulet (dir.), Université et territoires, Rennes, Presses Universitaires de Rennes, pp. 89104.

Milard Béatrice (2014), Increasingly international and visible research in the French provinces. Collaborations and citations of French chemists between 1985 and 2005, Conference Geography of Innovation, 23-25 janvier 2014, Utrecht University, Utrecht.

Milard Béatrice, Grossetti Michel, «L'évolution de la recherche scientifique dans les régions de Russie : déclin ou déconcentration? », M@ppemonde, n81, 2006. 
http://mappemonde.mgm.fr/num9/articles/art06105.html.

Naylor Simon, «Introduction: historical geographies of science-places, contexts, cartographies. », British Journal for the History of Science, Volume 38, n²1, 2005, pp.1-12.

Powell Richard C., «Learning from spaces of play: recording emotional practices in High Arctic environmental sciences », in Smith Mike, Davidson Joyce, Cameron Laura, Bondi Liz (Eds.), Emotion, Place and Culture, Ashgate, Farnham-Burlington VT, 2009.

Powell Richard C., "Geographies of science: histories, localities, practices, futures », Progress in Human Geography, Volume 31, n³, 2007, pp. 309-329.

Sealy Cordelia, 2004, "Globalization of science », Materials Today, Volume 7, Issue 5, Elsevier Ltd , mai 2004, p. 1

Shankar Ramamurti, «Globalization and Science: A Speeded-Up Virtuous Cycle », YaleGlobal, 28 mars 2003, http://yaleglobal.yale.edu/content/globalization-and-sciencespeeded-virtuous-cycle.

Shapin Steven, «Here and Everywhere: Sociology of Scientific Knowledge », Annual Review of Sociology, Volume 21, 1995, pp. 289-321

Sterlacchini Alessandro, 2008, «R\&D, higher education and regional growth: Uneven linkages among European regions », Research Policy, Volume 37, ${ }^{\circ}$ 6-7, 2008, pp. 1096110.

Taylor Peter J., Hoyler Michael, Evans David M., «A geohistorical study of "The Rise of Modern Science": mapping scientific practice through urban networks, 1500-1900», Minerva, n²6, 2008, pp.391-410.

Vassal Serge, L'Europe Des Universités, Caen, Editec, 1988.

Vinck Dominique, «The dynamics of Scientific Intellectuals Within the Integrative Trend in Europe: The Case of Co-operation Networks », in Elzinga Aant and Landström Catharina, Internationalism and Science, London, Taylor Graham, 1996, pp. 162-198.

Weiss Charles, «Science, technology and international relations », Technology in Society, Volume 27, n³, 2005, pp. 295-313.

Zitt Michel, Barré Rémi, Sigogneau Anne, Laville Françoise, «Territorial concentration and evolution of science and technology activities in the European Union: a descriptive analysis », Research Policy, Volume 28, n5, 1999, pp. 545-562. 